\title{
Reliability Evaluation of a Microgrid Considering Its Operating Condition
}

\author{
Xufeng $\mathrm{Xu}^{\dagger}$, Joydeep Mitra*, Tingting Wang** and Longhua Mu***
}

\begin{abstract}
Microgrids offer several reliability benefits, such as the improvement of load-point reliability and the opportunity for reliability-differentiated services. The primary goal of this work is to investigate the impacts of operating condition on the reliability index for microgrid system. It relies on a component failure rate model which quantifies the relationship between component failure rate and state variables. Some parameters involved are characterized by subjective uncertainty. Thus, fuzzy numbers are introduced to represent such parameters, and an optimization model based on Fuzzy Chance Constrained Programming (FCCP) is established for reliability index calculation. In addition, we present a hybrid algorithm which combines scenario enumeration and fuzzy simulation as a solution tool. The simulations in a microgrid test system show that reliability indices without considering operating condition can often prove to be optimistic. We also investigate two groups of situations, which include the different penetration levels of microsource and different confidence levels. The results support the necessity of considering operating condition for achieving accurate reliability evaluation.
\end{abstract}

Keywords: Chance constraint programming, Fuzzy number, Microgrid reliability, Microsource, Operating condition, Scenario

\section{Introduction}

Microgrid reliability evaluation is a well-established tool to measure the ability of a microgrid of withstanding random failure events in a long-term period. Reliability indices achieved are important parameters in microgrid planning and operation.

The potential benefit of microgrids in grid reliability improvement is an important advantage as pointed out in $[1,2]$. However, it is not always true that the reliability of power supply to all customers can be improved. While micro-source $(\mu \mathrm{S})$ may greatly increase power supply reliability for its owner, it may degrade the reliability index and the power quality of other customers on the same feeder [3].

Reliability indices are defined to measure the ability of continuous power supply to customers. There are significant differences between microgrid and distribution system in structure [4]. Thus, four special indices were introduced in [5] and another two were proposed in [6]. However, there is no uniform standard defined for microgrid reliability

$\dagger$ Corresponding Author: Department of Electrical Engineering, Tongji University, Shanghai, China and also Clemson University, Clemson, SC 29634 USA. (xfxu@tongji.edu.cn)

* Department of Electrical Engineering, Michigan State University, MI 48824 USA. (mitraj@msu.edu)

** American Electric Power, Columbus, OH 43215 USA. (twang@aep.com)

*** Department of Electrical Engineering, Tongji University, Shanghai, China. (lhmu@tongji.edu.cn)

Received: April 5, 2014; Accepted: September 21, 2015 indices so far. Many studies still follow reliability indices designed for the distribution system [7, 8]. In addition, two critical characteristics of microgrid are integrated $\mu \mathrm{Ss}$ and its autonomous control. Some publications were focusing on those two features [9, 10], A more recent study attempted to investigate the quantitative impact of cyber network failures on microgrid reliability [11].

In above approaches, the failure rate of component is measured by a constant value, which is provided by the manufacturer or obtained from the statistical data. The impacts of operating condition are ignored in the indices calculation. However, the analysis in [12] indicates that the increase in transfer capacity at line will amplify its failure probability. Furthermore, the probabilities of generator tripping and load shedding increase with the continued deviation of voltage. Hybrid condition-dependent outage models for transformer and transmission line were further proposed to include the impacts of various operating conditions [13].

Those previous results indicated that operating conditions have a significant impact on the reliability performance of transmission system only after multiple faults. The major reason for this phenomenon is that ' $\mathrm{N}-1$ ' ' security constraint is normally designed for transmission systems. However, no such preventive measure is normally designed to guarantee the security of microgrid. The purpose of this paper is to investigate the necessity of considering operating condition in the reliability evaluation of a microgrid.

This paper is organized as follows: in section 2, a component failure rate model is proposed to measure the 
relationship between component failure rate and state variables; in section 3 , an optimization model based on FCCP is established for reliability index calculation; in section 4, scenario technology is used to draw distinctions among the operation modes of microgrid; furthermore, a fuzzy simulation is adopted to handle fuzzy factors involved; in section 5, proposed model and approach are tested in a small microgrid system; a summary is given in section 6 .

\section{Failure Rate Modeling for Feeders}

Generally, the failure rate of a feeder is measured by its annual statistical result based on an assumption that this feeder is operating under its rated condition. However, the microgrid has versatile operating conditions due to its multiple operation modes and integrated small-scale $\mu \mathrm{Ss}$. Abnormal operating conditions occasionally happen, such as overcurrent, overvoltage and under-voltage. Furthermore, bi-directional power flows may occur in microgrids, which is different from the conventional distribution network. Thus, there is a significant necessary to integrate the operating condition in evaluating feeder failure rate.

In this study, voltage $U$ and current $I$ are selected to represent the state variables of a feeder. Let $\lambda_{0}$ be the longterm statistical result of the failure rate and $\lambda$ be the one considering operating condition. $\lambda$ could be evaluated by two introduced coefficients $h(U)$ and $h(I)$,

$$
\lambda=\max \{h(U), h(I)\} \cdot \lambda_{0}
$$

\subsection{Overvoltage and undervoltage coefficients}

The variation in voltage magnitude is a significant parameter in the measurement of power quality. According to the criterion of power supply in China, the allowable variation $\varepsilon$ is set to $5 \%$ or $7 \%$ (the former has been adopted in this study). The maximum variation, which is represented by $\delta$, is regarded to be related to customer demand on power quality or the setting value of protective relay. Thus, a piecewise function $h(U)$ is designed to describe the voltage related coefficients, as shown in Fig. 1 and explained as follows.

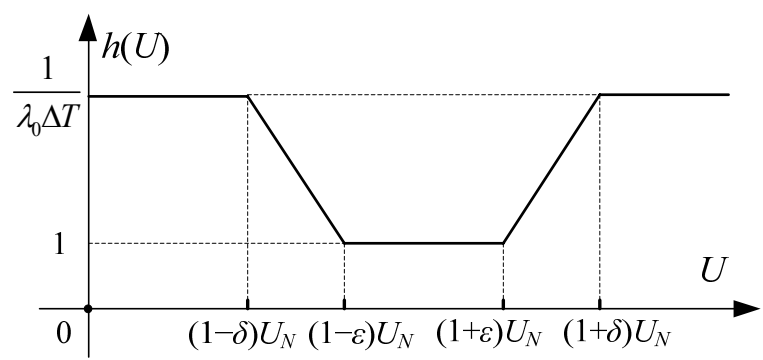

Fig. 1. The impact of voltage on short-term failure rate
1) $(1-\varepsilon) U_{N} \leq U \leq(1+\varepsilon) U_{N}$. Here, $U_{N}$ is the rated voltage. In the normal condition, conventional failure rate $\lambda_{0}$ is deployed to $\lambda$, or $h(U)=1$.

2) $U \leq(1-\delta) U_{N}$ or $U \geq(1+\delta) U_{N}$. Protective device should operate since the state variable exceeds its allowable limits. The feeder will be loss of power until fault recovery. If the short-term period $\Delta T$ is comparable to the repair time of the feeder, $\lambda$ can be approximated by $1 / \Delta T[14]$. The expression of $h(U)$ is

$$
h(U)=1 /\left(\lambda_{0} \cdot \Delta T\right)
$$

3) $(1-\delta) U_{N} \leq U \leq(1-\varepsilon) U_{N}$. A linear function is deployed to fit the relationship between $h(U)$ and $U$,

$$
h(U)=\frac{\left(1+\lambda_{0} \Delta T \delta-\varepsilon-\lambda_{0} \Delta T\right) U_{N}+\left(\lambda_{0} \Delta T-1\right) U}{\lambda_{0} \Delta T U_{N}(\delta-\varepsilon)}
$$

4) $(1-\varepsilon) U_{N} \leq U \leq(1+\delta) U_{N}$. A linear function is also chosen,

$$
h(U)=\frac{\left(\lambda_{0} \Delta T+\lambda_{0} \Delta T \delta-1-\varepsilon\right) U_{N}+\left(1-\lambda_{0} \Delta T\right) U}{\lambda_{0} \Delta T U_{N}(\delta-\varepsilon)}
$$

\subsection{Overcurrent coefficient}

$h(I)$ is analyzed in a similar way. Assuming $I_{N}$ is normal rating value and $I_{S}$ is short-time rating value, which are defined in [15], the expression of $h(I)$ is explained in (5) and Fig. 2.

$$
h(I)=\left\{\begin{array}{cc}
1, & 0 \leq|I| \leq I_{N} \\
\frac{\lambda_{0} \Delta T I_{S}-I_{N}+I\left(1-\lambda_{0} \Delta T\right)}{\lambda_{0} \Delta T\left(I_{S}-I_{N}\right)}, & I_{N}<|I| \leq I_{S} \\
\frac{1}{\lambda_{0} \Delta T}, & |I|>I_{S}
\end{array}\right.
$$

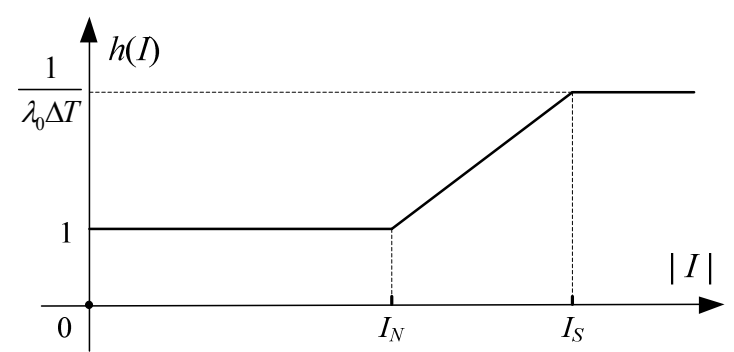

Fig. 2. The impact of current on short-term failure rate

\section{Short-time Reliability Evaluation Model.}

Scenario technology is introduced to handle the stochastic 
environment related to operating environment. In addition, parameters involved in failure rate models are simulated using fuzzy numbers and investigated based on credibility theory.

\subsection{Operating condition related reliability indices}

Many $\mu \mathrm{Ss}$ are fundamentally dependent on weather condition. For example, photovoltaic arrays rely on illumination and its ambient temperature must be in a special range for normal operation. Similarly, wind turbine generator delivers power only within a special speed limit. Although weather conditions could not be forecasted accurately with current weather forecasting technology, annual meteorological data follows certain regularity in the location of microgrid. Those meteorological data including ambient temperature $E(t)$, illumination $S(t)$ and wind speed $V(t)$ can be obtained from local meteorological department.

Each combination of $E(t), S(t)$ and $V(t)$ is defined as a scenario represented by $\sigma$, as shown in (6). Scenario $\sigma$ is distinguished by the values of three parameters.

$$
\sigma=\{E(t), S(t), V(t)\}
$$

Considering those three parameters are continuous variables, scenario number is a tremendous value. Therefore, meteorological data are discretized in the following way: the same value is deployed for all sampling parameters at the range within an individual step. Assuming $n\left(\sigma_{e}\right)$ is the number of scenario $\sigma_{e}, N_{\sigma}$ is scenario types and $f$ is sampling frequency, their relationship is described in (7).

$$
\sum_{e=1}^{N_{\sigma}} n\left(\sigma_{e}\right)=8760 / f
$$

Subsequently, six reliability indices defined for distribution system [16] are modified to evaluate the reliability of microgrid considering operating conditions,

$$
\begin{aligned}
\mathrm{SAIFI} & =\sum_{e=1}^{N_{\sigma}}\left(\frac{n\left(\sigma_{e}\right) \cdot f}{8760} \cdot \frac{\sum_{i=1}^{N}\left(\xi_{i}\left(\sigma_{e}\right) \cdot m_{i}\right)}{\sum_{i=1}^{N} m_{i}}\right) \\
\mathrm{SAIDI} & =\sum_{e=1}^{N_{\sigma}}\left(\frac{n\left(\sigma_{e}\right) \cdot f}{8760} \cdot \frac{\sum_{i=1}^{N}\left(\omega_{i}\left(\sigma_{e}\right) \cdot m_{i}\right)}{\sum_{i=1}^{N} m_{i}}\right) \\
\mathrm{CAIDI} & =\sum_{e=1}^{N_{\sigma}}\left(\frac{n\left(\sigma_{e}\right) \cdot f}{8760} \cdot \frac{\sum_{i=1}^{N}\left(\omega_{i}\left(\sigma_{e}\right) \cdot m_{i}\right)}{\sum_{i=1}^{N}\left(\xi_{i}\left(\sigma_{e}\right) \cdot m_{i}\right)}\right) \\
\mathrm{ASAI} & =\sum_{e=1}^{N_{\sigma}}\left(\frac{n\left(\sigma_{e}\right) f}{8760} \frac{8760 \times \sum_{i=1}^{N} m_{i}-\sum_{i=1}^{N}\left(\omega_{i}\left(\sigma_{e}\right) \cdot m_{i}\right)}{8760 \times \sum_{i=1}^{N} m_{i}}\right)
\end{aligned}
$$

$$
\begin{aligned}
\mathrm{ENS} & =\sum_{e=1}^{N_{\sigma}}\left(\frac{n\left(\sigma_{e}\right) \cdot f}{8760} \cdot \sum_{i=1}^{N}\left(D_{i}\left(\sigma_{e}\right) \cdot \omega_{i}\left(\sigma_{e}\right)\right)\right) \\
\mathrm{AENS} & =\sum_{e=1}^{N_{\sigma}}\left(\frac{n\left(\sigma_{e}\right) \cdot f}{8760} \cdot \frac{\sum_{i=1}^{N}\left(D_{i}\left(\sigma_{e}\right) \cdot \omega_{i}\left(\sigma_{e}\right)\right)}{\sum_{i=1}^{N} m_{i}}\right)
\end{aligned}
$$

where, $N$ is the number of load points; $\xi_{i}\left(\sigma_{e}\right)$ is average failure rate of $i$ th load point $\mathrm{LP}_{i}$ under scenario $\sigma_{e} ; \omega_{i}\left(\sigma_{e}\right)$ is average outage time of $\mathrm{LP}_{i} ; m_{i}$ is the number of customer at $\mathrm{LP}_{i} ; D_{i}\left(\sigma_{e}\right)$ is load demand at $\mathrm{LP}_{i}$.

\subsection{Optimization model}

Although factors including $\varepsilon, \delta, I_{N}$ and $I_{S}$ rely on the property of a feeder, human experience and decision always play an important role in determining those parameters. In addition, some operating constrains are mathematically soft constraints, such as $U \leq(1+\varepsilon) U_{N}$ and $I \leq I_{N}$. Thus, those parameters are subject to a subjective uncertainty and they are represented by a fuzzy variable $\tilde{\boldsymbol{\rho}}$ in this study. Assuming $\tilde{\boldsymbol{\rho}}$ is determined by a triangular fuzzy value $\left(\rho_{1}, \rho_{2}, \rho_{3}\right)$, its membership function $\mu(\tilde{\boldsymbol{\rho}})$ is shown in Fig. 3

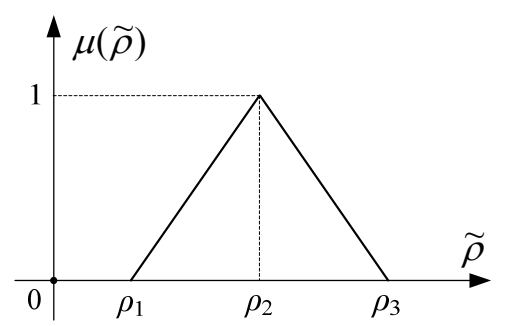

Fig. 3. The membership of fuzzy number

According to the above analysis, each defined reliability index $F_{k}(k=1,2, \ldots, 6)$ is also a fuzzy value and it is handled based on credibility theory [17]. In each scenario, short-time reliability indices are calculated by an optimization model based on FCCP. The objective function is chosen as the $\gamma_{k}$-pessimistic value of reliability index $F_{k}$. It is desired that constraint ' $F_{k} \leq F_{k, M}$ ' must hold at a confidence level $\gamma_{k}$.

$$
\text { Minimize } F_{k, M}
$$

Subject to:

$$
\begin{gathered}
\operatorname{Cr}\left\{F_{k}(\boldsymbol{I}, \boldsymbol{U}, \tilde{\boldsymbol{\rho}}) \leq F_{k, M}\right\} \geq \gamma_{k} \\
G_{P, i}-D_{P, i}-\sum_{j \in \boldsymbol{\Lambda}_{i}} U_{i} U_{j}\left(g_{i j} \cos \theta_{i j}+b_{i j} \sin \theta_{i j}\right)=0 \\
G_{Q, i}-D_{Q, i}-\sum_{j \in \boldsymbol{\Lambda}_{i}} U_{i} U_{j}\left(g_{i j} \sin \theta_{i j}-b_{i j} \cos \theta_{i j}\right)=0 \\
|I| \leq \tilde{I}_{S} \\
(1-\tilde{\delta}) U_{N} \leq U \leq(1+\tilde{\delta}) U_{N}
\end{gathered}
$$


where,
$\boldsymbol{\Lambda}_{i}$ is the set of nodes which are connected to node $i$;
$\theta_{i j}$ is the phase angle difference of feeder $i j$;
$g_{i j}$ is the resistance of feeder $i j$ and $b_{i j}$ is reactance;
$G_{P, i} / G_{Q, i}$ is the power output of $\mu \mathrm{S}$;
$D_{P, i} / D_{Q, i}$ is active/reactive load demand at node $i$;

Constraints (16) and (17) represent power flow equations; constraints (18) and (19) set limitations for state variables. In fuzzy chance constraint (15), credibility measure ' $\operatorname{Cr}\left\{F_{k} \leq F_{k, M}\right\}$ ' indicates the satisfaction that $F_{k}$ is not larger than $F_{k, M}$. $\mathrm{Cr}\left\{F_{k} \leq F_{k, M}\right\}$ can be calculated by possibility measure 'Pos $\left\{F_{k} \leq F_{k, M}\right\}$ ' and necessity measure 'Nec $\left\{F_{k} \leq F_{k, M}\right\}$ ',

$$
\operatorname{Cr}\left\{F_{k} \leq F_{k, M}\right\}=\left(\operatorname{Pos}\left\{F_{k} \leq F_{k, M}\right\}+\operatorname{Nec}\left\{F_{k} \leq F_{k, M}\right\}\right) / 2
$$

\section{Solution Process}

A failure mode and effects analysis based hybrid approach is proposed to calculate reliability indices.

\subsection{Load point classification}

All feeder failures contributed to the reliability indices of load points are enumerated. Protective devices including relays and fuses then operate to limit failure impacts in a certain area. Subsequently, the whole system is divided into several subsystems according to the switch statuses of breaker, disconnector and tie switch. For a good explanation, load points $\operatorname{LP}_{i}(i=1,2, \ldots, N)$ under $l$ th fault $(l=1,2, \ldots, M, M$ is the feeder number $)$ are classified into four classes, as given in Table 1.

Class A: this class is not connected to any backup power sources and they will be loss of power until fault recovery. Its outage time $r_{i l}$ is depended on mean repair time $t_{r}$.

Class B: no influence.

Class C: the load recovery of this class is after disconnector operation. $r_{i l}$ is fault isolation time $t_{d}$.

Class D: $r_{i l}$ relies on available power from $\mu \mathrm{S}$ or tie switch with surplus capacity. $r_{i l}$ is the sum of $t_{d}$ and load transfer time $t_{c}$. In some cases, only $y \%$ of load demand can be restored for a load point; and then $r_{i l}$ is corrected by $t^{\prime}$,

Table 1. Classification of load points

\begin{tabular}{c|c|c|c}
\hline Class & Impacts & Energy Sources & $r_{i l}$ \\
\hline $\mathrm{A}$ & Outage & No & $t_{r}$ \\
\hline $\mathrm{B}$ & No influence & Feeder or $\mu \mathrm{S}$ & 0 \\
\hline $\mathrm{C}$ & Outage in a short time & Feeder or $\mu \mathrm{S}$ & $t_{d}$ \\
\hline $\mathrm{D}$ & Outage in a short time & Backup power supply & $t_{d}+t_{c}$ \\
\hline
\end{tabular}

$$
t^{\prime}=(1-y \%) \cdot t_{r}+y \% \cdot t_{d}+y \% \cdot t_{c}
$$

This classification is based on a premise that a fault has happened. $\mathrm{LP}_{i}$ may be categorized into different classes under different faults.

Four classes of load points have different priorities in load recovery. Breadth-first search strategy is adopted to determine load restore sequence. Load point with the closest electrical distance from $\mu \mathrm{Ss}$ is recovered first. If more than one load points have the same electrical distance, load importance is used as a priority rule.

\subsection{Calculation of customer reliability indices}

Three hypotheses are first introduced to evaluate customer reliability indices:

1) Circuit breakers are installed only in the power source end of main feeder or access points of $\mu \mathrm{Ss}$;

2) Disconnectors are allocated in all subsections of main feeder and fuses are installed in all lateral distributors;

3) Fuse operation is instantaneous and accurate.

For $\mathrm{LP}_{i}$, its average failure rate $\xi_{i}$, average outage time $\omega_{i}$ and average annual outage time $T_{i}$ are calculated according to $\lambda_{l}$ and $r_{i l}$ analyzed above, as shown in (22), (23) and (24), respectively. Here, $\operatorname{sgn}(\cdot)$ is the signum function in mathematics. Subsequently, system reliability indices are evaluated according to their definitions.

$$
\begin{gathered}
\xi_{i}=\sum_{l=1}^{M} \lambda_{l} \cdot \operatorname{sgn}\left(r_{i l}\right) \\
\omega_{i}=\sum_{l=1}^{M}\left(r_{i l} \cdot \lambda_{l}\right) \\
T_{i}=\omega_{i} / \xi_{i}
\end{gathered}
$$

Taking $\mathrm{LP}_{1}$ in Fig. 4 as an example, the evaluation process of its reliability indices is summarized in Table 2 and analyzed as follows,

1) If a fault occurs at main feeder $L_{i j}, \mathrm{LP}_{1}$ is classified as class $\mathrm{C}$ or $\mathrm{D}$ and $r_{1 l}$ is equal to $t_{d}$ or ' $t_{d}+t_{c}$ '.

2) If a fault occurs at main feeder $L_{j k}, \mathrm{LP}_{1}$ belongs to class $\mathrm{C}$ and $r_{1 l}$ equals ' $t_{d}$ '.

3) If a fault occurs at lateral distributor $l_{1}, \mathrm{LP}_{1}$ is class A. It will be recovered only after $l_{1}$ is repaired.

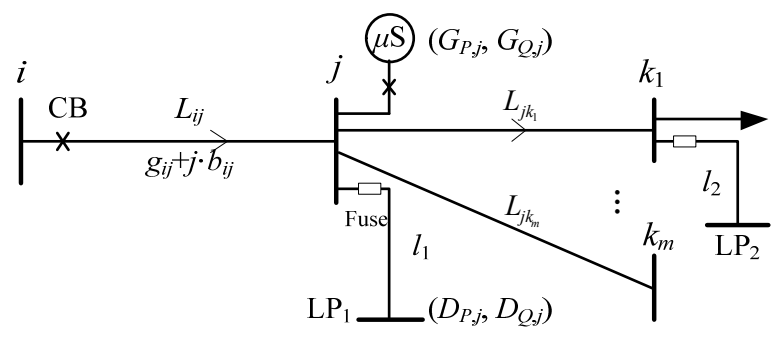

Fig. 4. A part of microgrid system 
Table 2. Reliability indices of $\mathrm{LP}_{1}$

\begin{tabular}{c|c|c|c}
\hline \multirow{2}{*}{$\mathrm{LP}_{1}$} & \multicolumn{2}{|c|}{ Main feeder } & Lateral distributor \\
\cline { 2 - 4 }$\xi_{1}(f / y r)$ & $L_{i j}$ & $L_{j k_{c}}$ & $l_{1}$ \\
\hline$r_{1 l}$ (hours) & $\lambda$ & $\sum_{c=1}^{m} \lambda$ & $\lambda$ \\
\hline$\omega_{1}$ (hours $\left./ y r\right)$ & $\lambda \cdot t_{d}$ or $\lambda \cdot\left(t_{d}+t_{c}\right)$ & $\sum_{c=1}^{m} \lambda \cdot t_{d}$ & $\lambda \cdot t_{r}$ \\
\hline
\end{tabular}

\subsection{Fuzzy simulation for $\gamma_{k}$-pessimistic value $F_{k, M}$}

Fuzzy simulation is used to approximate the fuzzy chance constraint ' $\operatorname{Cr}\left\{F_{k} \leq F_{k, M}\right\} \geq \gamma_{k}$ ' in the optimization model. A monotonous function $L\left(f_{k}\right)$ is introduced for the calculation of $F_{k, M}$, as in (25). A bisection search is employed to find the maximal value $f_{k}$ which is used to estimate $F_{k, M}$.

$$
\begin{aligned}
L\left(f_{k}\right)=\frac{1}{2}\left(\max _{1 \leq m \leq N_{M}}\left\{v_{m} \mid F_{k}\left(\rho_{m}\right) \leq f_{k}\right\}\right. \\
\left.\quad+\min _{1 \leq m \leq N_{M}}\left\{1-v_{m} \mid F_{k}\left(\rho_{m}\right)>f_{k}\right\}\right)
\end{aligned}
$$

The procedure of fuzzy simulation is shown in Fig. 5 and main steps are given as follows.

Step 1) Generate a fuzzy vector $\boldsymbol{\rho}_{m}=\left(\rho_{1}, \rho_{2}, \cdots \rho_{N_{F}}\right)$ from credibility space randomly, $m=1,2, \ldots N_{M}$ ( $N_{F}$ is the number of fuzzy variables and $N_{M}$ is

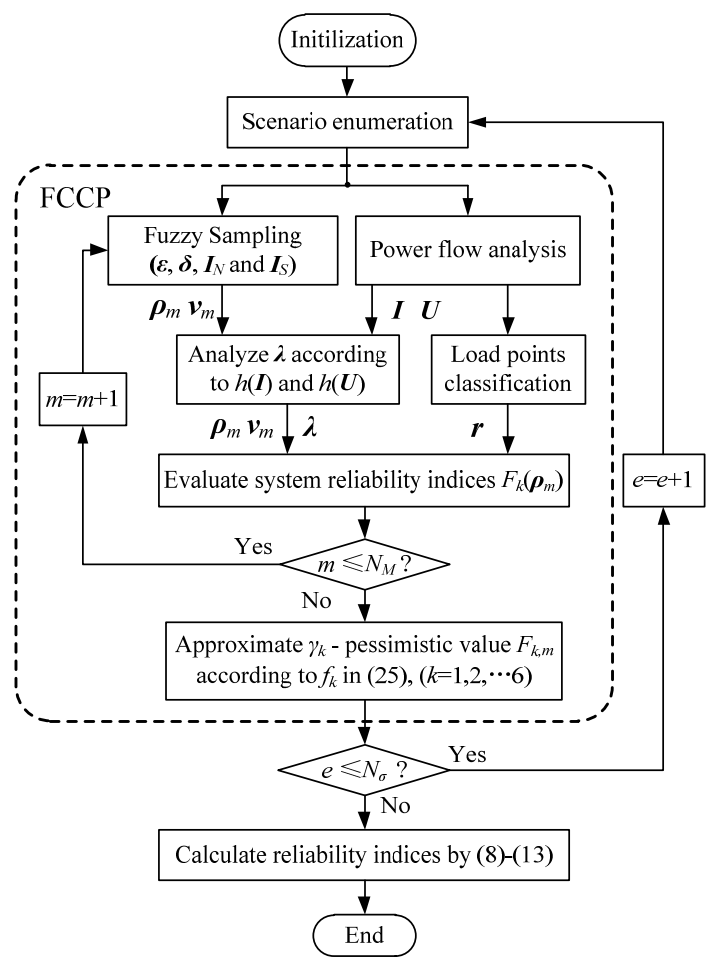

Fig. 5. The flowchart of approach fuzzy sampling number);

Step 2) Calculate a membership vector $\boldsymbol{v}_{m}=\mu\left(\boldsymbol{\rho}_{m}\right)$;

Step 3) Find $f_{k}$ satisfied the inequality constraint ' $L\left(f_{k}\right) \geq$ $\gamma_{k}^{\prime}$;

Step 4) Return $f_{k}$ as the estimation value of $F_{k, M}$.

Step 5) Repeat steps $1 \sim 4 N_{\sigma}$ times.

Step 6) Calculate the reliability indices according to (8)(13).

\section{Case Study}

The microgrid system proposed in [18] is chosen as a test system and some modifications were made by the authors in [14]. This system comprises 11 nodes, 12 main feeders and 10 load points (LP). $U_{N}$ is $400 \mathrm{~V}$ and $\delta$ is set to $10 \%$. This study generates four kinds of fuzzy parameters by multiplying a triangular fuzzy factor $(0.9,1.0,1.1)$.

\subsection{Comparisons of reliability indices}

A simulation was implemented to compare the proposed approach with the conventional approach, in which $\lambda_{0}$ is used to assess the failure rate of each feeder. The reliability performances of feeders, customers and the system are presented as follows.

\subsubsection{Feeder reliability}

The failure rates of feeders except for two tie lines are compared in Fig. 6. It can be noticed that feeder failure rates increase remarkably in the proposed approach. For example, maximalratio of two cases is close to 2.86 times at No. 8 feeder. In addition, the failure rates of No.1 and No.2 feeders have two biggest values due to the fact that they are two longest feeders. Some effective measures are needed on them to improve their reliability indices.

\subsubsection{Customer reliability}

Average annual outage times of load points are compared

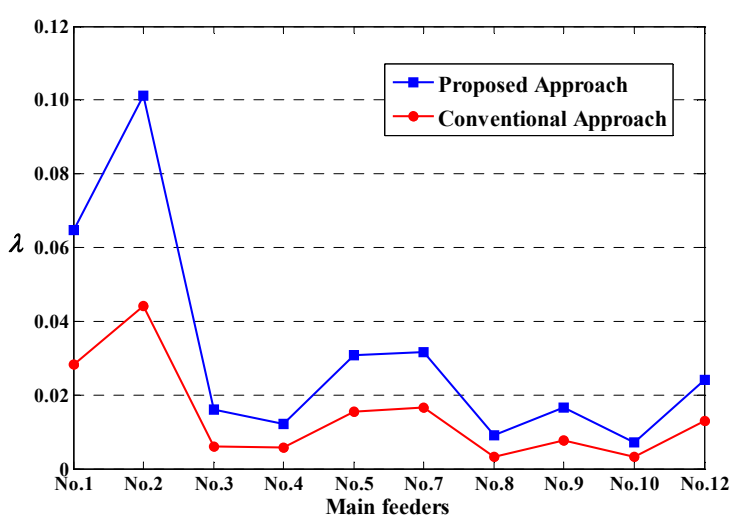

Fig. 6. The comparisons of feeder failure rate 


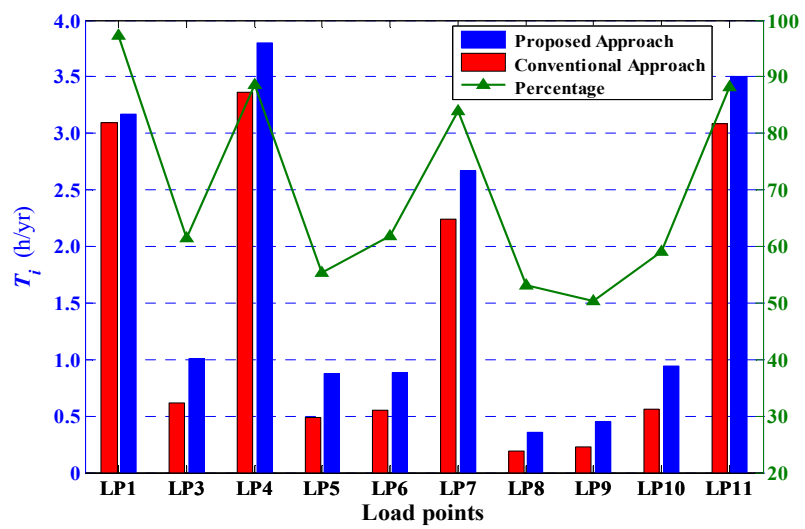

Fig. 7. Average annual outage time of customers

in Fig. 7. Load points including LP3, LP5, LP6, LP8, LP9 and LP10 have relatively low values. It is because there are $\mu \mathrm{Ss}$ in or near those load points. The proportions of the results achieved by two approaches are also indicated in Fig. 7. Load points far away from $\mu$ Ss are influenced much less.

On the other hand, $\mu \mathrm{S}$ development will not help customers to decrease their failure rate of power supply. However, it can shorten outage time and recover power as soon as possible.

\subsubsection{System Reliability}

Six indices defined in $(8) \sim(13)$ are compared in Table 3. In the conventional approach, it is assumed that all feeders are operating under normal conditions. Therefore, only statistical results of feeder failure rates are used in the evaluation process. The achieved reliability indices turn out to be optimistic results. It can be found in Table 3 that the most significance difference between two cases is SAIFI. Its deviation reaches more than $50 \%$.

Table 3. Reliability indices of microgrid system

\begin{tabular}{c|c|c|c}
\hline Reliability indices & $\begin{array}{c}\text { Proposed } \\
\text { approach }\end{array}$ & $\begin{array}{c}\text { Conventional } \\
\text { approach }\end{array}$ & Deviation \\
\hline SAIFI (int/cus-yr) & 0.3530 & 0.1684 & $52.30 \%$ \\
\hline SAIDI ( $h r / c u s-y r)$ & 1.8673 & 1.4896 & $20.23 \%$ \\
\hline CAIDI (hr/int) & 6.6118 & 8.8456 & $33.78 \%$ \\
\hline ASAI & 0.9998 & 0.9998 & $0.00 \%$ \\
\hline ENS (MWh/yr) & 90.3919 & 72.4992 & $19.79 \%$ \\
\hline AENS (MWh/cus-yr) & 0.2205 & 0.1768 & $19.79 \%$ \\
\hline
\end{tabular}

\subsection{Impacts of operating condition}

Reliability indices related to system and customers are calculated based on the failure rates of feeders; therefore, this section only studies the relationship between operating condition and feeder failure rate. In Fig. 8, the horizontal axis shows main feeders; the vertical axis shows load rate $\left(|I| / I_{N}\right)$ and voltage deviation $\Delta U\left(\Delta U=\left|U-U_{N}\right|\right)$. Bar graph represents outage rate per unit length, which is different from the curves in Fig. 6.

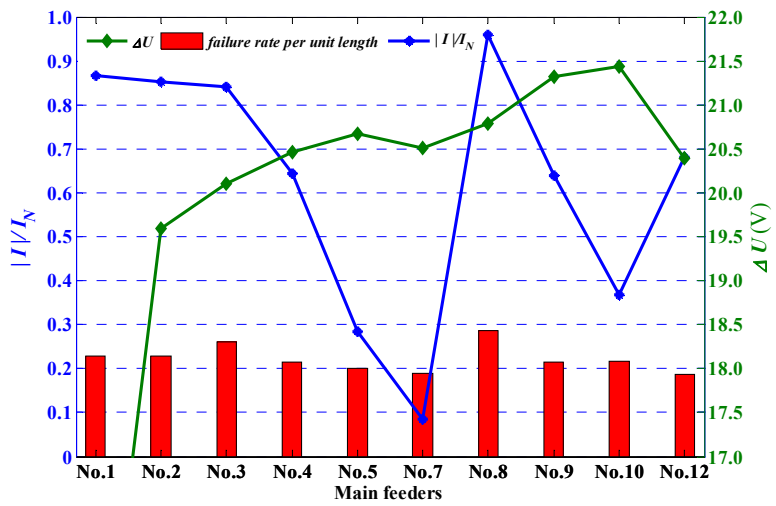

Fig. 8. Operating conditions and failure rates of main feeders

Two conclusions are deduced from Fig. 8: (1) The outage rates of feeders which are close to main power source or the slack bus are more sensitive to load rate than voltage deviation, exemplified by four feeders including No. 1, No. 2, No.3 and No.8. (2) Feeders far away from power sources are more likely to be influenced by voltage deviation, such as, No. 5, No. 7, No. 9 and No. 10.

\subsection{Reliability indices of microgrid with different penetrations}

$\mu$ Ss are normally installed near to load points; therefore, they can potentially improve customer and system reliability indices. This section investigates system reliability indices and the penetration level of $\mu \mathrm{S}$. A penetration factor $\eta$ is defined as,

$$
\eta\left(\sigma_{e}\right)=\frac{G\left(\sigma_{e}\right)}{D\left(\sigma_{e}\right)} \times 100 \%
$$

where $G\left(\sigma_{e}\right)$ is total power output of $\mu \mathrm{S}$ in scenario $\sigma_{e}$ and $D\left(\sigma_{e}\right)$ is total load demand.

The simulation of SAIFI is shown in Fig. 9. SAIFI could not be improved when $\eta$ is larger than 0.4528. In other words, there is a limitation for SAIFI improvement through $\mu \mathrm{S}$ installment. This conclusion holds a significant value in

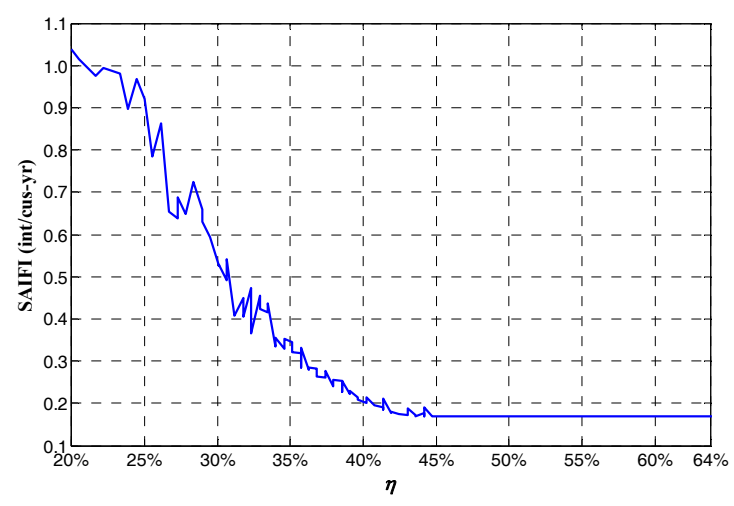

Fig. 9. SAIFI under different penetrations 


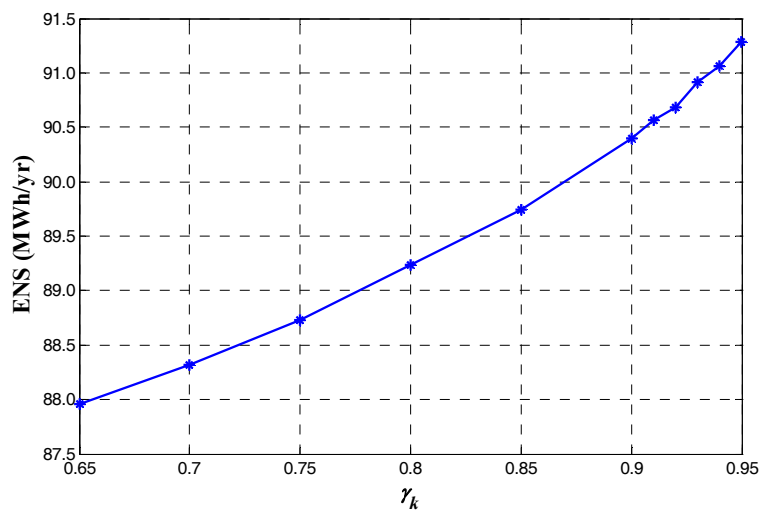

Fig. 10. $\gamma_{k}$-pessimistic values of ENS

microgrid planning. Furthermore, $\eta$ is not the only reason for SAIFI variation. Small margin fluctuations in the curve are jointly caused by the types or locations of $\mu \mathrm{S}$.

\subsubsection{Pessimistic values under different confidence levels}

The confidence level is one of the most important parameters in the FCCP based model. Fig. 10 shows that $\gamma_{k}$-pessimistic value $F_{k, M}$ is monotonic increasing along with confidence level $\gamma_{k}$. The larger $\gamma_{k}$ is, the larger value $F_{k, M}$ is needed to satisfy the inequality ' $F_{k} \leq F_{k, M}$ '. Thus, only a small reliability index can be expected when a large value of confidence level is demanded.

\section{Conclusion}

This paper investigates the impacts of operating condition on microgrid reliability. The simulation on a microgrid test system demonstrates the necessity for analyzing component failure rate in the microgrid reliability evaluation. It also shows that customers in a microgrid could not have the same reliability benefit of power supply at different locations and periods.

\section{Acknowledgements}

This work is supported by National Natural Science Foundation of China (51407128).

\section{References}

[1] P. M. Costa and M. A. Matos, "Assessing the contribution of microgrids to the reliability of distribution networks," Elect. Power Syst. Res., vol. 79, no. 2, pp.382-389, 2009.

[2] S. Kennedy, "Reliability evaluation of islanded microgrids with stochastic distributed generation," presented at Power Eng. Soc. Gen. Meeting, Calgary,
AB, Canada, July 26-30, 2009.

[3] R. C. Dugan and T. E. McDermott, "Operating conflicts for distributed generation on distribution system," presented at Rural Electric Power Conference, Little Rock, Arkansas, USA, April 29-May 1, 2001.

[4] Y. Luo, L. J. Wang, G. Zhu, and G. Wang, "Network analysis and algorithm of microgrid reliability assessment," presented at Asia-Pacific Power and Energy Engineering Conference (APPEEC), Chengdu, China, March 28-31, 2010.

[5] R. Yokoyama, T. Niimura, and N. Saito, "Modeling and evaluation of supply reliability of microgrids including PV and wind power," presented at Power and Energy Society General Meeting - Conversion and Delivery of Electrical Energy in the 21st Century, Pittsburgh, USA, July 20-24, 2008.

[6] Z. Li, Y. Yuan, and F. Li, "Evaluating the reliability of islanded microgrid in an emergency mode," presented at 45th International Universities Power Engineering Conference (UPEC), Aug. 31 -Sep. 3, 2010.

[7] I. S. Bae, and J. O. Kim, "Reliability evaluation of distributed generation based on operation mode," IEEE Trans. Power Syst., vol. 22, no. 2, pp. 785-790, May, 2007.

[8] I. S. Bae, and J. O. Kim, "Reliability evaluation of customers in a microgrid," IEEE Trans. Power Syst., vol. 23, no. 3, pp. 1416-1422, Aug. 2008.

[9] S. Kennedy, and M. M. Marden, "Reliability of islanded microgrids with stochastic generation and prioritized load," presented at IEEE Bucharest Power Tech Conference, Bucharest, Romania, June 28-July 2, 2009.

[10] Z. Bie, P. Zhang, G. Li, and B. Hua, M. Meehan and $\mathrm{X}$. Wang, "Reliability evaluation of active distribution systems including microgrids," IEEE Trans. Power Syst., vol. 27, no. 4, pp. 1-9, Nov. 2012.

[11] B. Falahati, Y. Fu, and L. Wu, "Reliability assessment of smart grid considering direct cyber-power interdepend- dencies," IEEE Trans. Smart Grid, vol. 3, no. 3, pp. 1-10, Sep. 2012.

[12] Y. Z. Sun, L. Cheng, H. T. Liu, and S. He. "Power system operational reliability evaluation based on real-time operating state," presented at the $7^{\text {th }}$ International Power Engineering Conference, pp. 722727, Nov. 29-Dec. 2, 2005.

[13] J. He, Y. Z. Sun, P. Wang, and L. Cheng. "A hybrid conditions- dependent outage model of a transformer in reliability evaluation," IEEE Trans. Power Deli., vol. 24, no. 4, pp. 2025-2033, Oct. 2009.

[14] X. Xu, J. Mitra, T. Wang, and L. Mu, "Evaluation of operational reliability of a microgrid using a shortterm outage model," IEEE Trans. Power Syst., vol.29, no.5, pp. 2238-2247, Sep. 2014.

[15] P. M. Subcommittee, "IEEE Reliability Test System," 
IEEE Trans. Power App. Syst., vol. PAS-98, no. 6, pp. 2047-2054, Nov./Dec., 1979.

[16] R. Billinton, and R. N. Allan, Reliability evaluation of power systems (Edition 2), New York, NY: Plenum Press, 1996.

[17] B. Liu, Theory and Practice of Uncertain Programming (Edition 2), Berlin, Germany: SpringerVerlag, 2009.

[18] K. Rudion, A. Orths, Z. A. Styczynski, and K. Strunz, "Design of benchmark of medium voltage distribution network for investigation of DG integration," in proc. Power Engineering Society General Meeting, Montreal, QC, Canada, Sep. 2006.

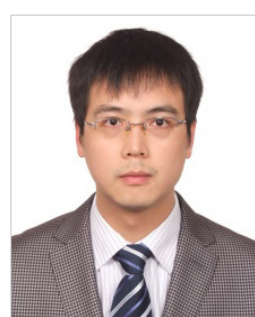

Xufeng Xu He received the B.E. degree and the Ph.D. degree in electrical engineering from Zhejiang University, Hangzhou, China. His research interests include microgrid reliability, power system planning, and optimization algorithms.

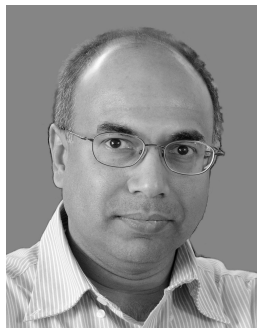

Joydeep Mitra $\mathrm{He}$ received the B. Tech. (Hons.) degrees in electrical engineering from Indian Institute of Technology, Kharagpur, India, and the Ph.D. degree in electrical engineering from Texas A\&M University, College Station, TX, USA. His research interests include power system reliability, distributed energy resources, and power system planning.

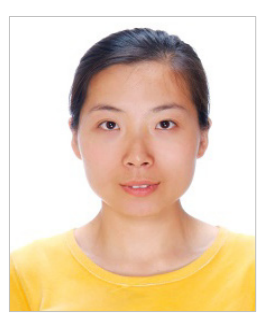

Tingting Wang She received the Ph.D. degree in electrical engineering from Clemson University, Clemson, USA. Her research interests are renewable energy integration, power system transient and electricity market.

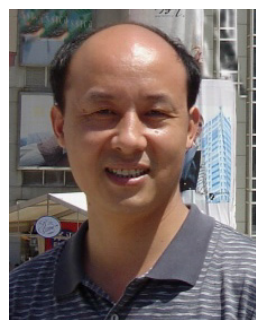

Longhua Mu He received the B.E., M.E. and Ph.D. degrees in electrical engineering from China University of Mining and Technology, Xuzhou, China. His current research interests include protective relaying of power system, power quality and microgrid control. 